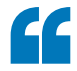

$\mathrm{Mx}$ 1-Cre; HUe mice revealed an unexpected consistency in the behaviour of HSC clones

HAEMATOPOIESIS

\title{
HSC function determined by epigenetic memory
}

Haematopoietic stem cells (HSCs) are functionally heterogeneous, having distinctive behaviours in terms of proliferative potential and lineage bias. They are often thought of as 'plastic' cells that can adapt their function to the needs of the organism, in terms of homeostasis or the response to stress, by responding to environmental factors. However, the results of a new study published in Cell call these assumptions into question by showing that individual HSC clones have a stereotypical behaviour that is determined by their retained epigenetic state.

Previous studies of HSC heterogeneity have used ex vivo DNA barcoding or retrotransposon tagging of endogenous cells. As the analysis of HSCs labelled by these techniques requires cell destruction, they cannot be transplanted into new hosts for long-term study. To overcome this problem, Scadden and colleagues created a new mouse strain (termed HUe) in which multiple
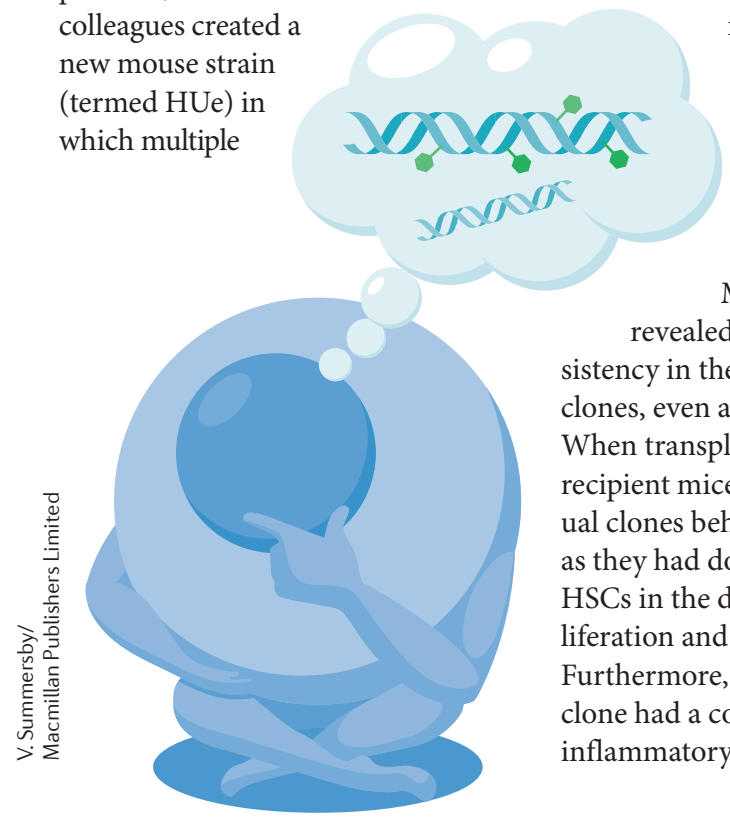

fluorescent protein-encoding genes can be recombined stochastically, under the control of Cre recombinase, to label cells with a large range of possible colours (theoretically greater than $1,000 \mathrm{col}$ ours). HUe mice were crossed with interferon-inducible Mx1-Cre mice (generating $\mathrm{Mx1-Cre;HUe} \mathrm{mice)} \mathrm{to}$ allow the labelling of endogenous haematopoietic cells to be induced using polyinosinic-polycytidylic acid (poly(I:C)).

Poly(I:C)-induced Mx1-Cre;HUe mice contained clusters of HSCs of the same colour that represented clonal descendants, and these colour signatures were stably retained in vitro and in vivo. Studying the dynamics of normal haematopoiesis in these mice showed that a few major HSC clones persist over the course of many months, whereas other clones expand, disappear or newly emerge, which is consistent with other recent studies showing that the majority of the haematopoietic system is maintained by just a few out of many clones.

However, the Mx1-Cre;HUe mice

across all recipient mice, despite there being interclonal variation in the response. These results indicate that the in vivo behaviour of HSCs is determined in a cell-autonomous manner rather than by external factors, which would presumably differ between recipients.

Further study correlating the enhancer DNA methylation state and chromatin accessibility of independent HSC clones with their function showed that the clonal behaviour of HSCs is determined by epigenetic mechanisms. For example, whereas the Cohort2.G HSC clone contributed to both myeloid and lymphoid lineages, the Cohort2.P HSC clone mainly contributed to the lymphoid lineage. Correspondingly, the Cohort 2.G clone had lower levels of DNA methylation and higher levels of chromatin accessibility in enhancer regions specific to common myeloid progenitors.

Thus, the authors suggest that the function of individual HSC clones is determined by an epigenetic memory that is acquired early during development of the haematopoietic system and that is retained in different hosts and under different environmental conditions. The adaptive response of the HSC pool to various stressors may therefore reflect the differential activation of specific clones rather than any change in the behaviour of individual clones.

Kirsty Minton

This article is modified from the original in Nature Rev. Mol. Cell Biol. (doi:10.1038/nrm.2016.161). as they had done as endogenous HSCs in the donor in terms of proliferation and lineage commitment. Furthermore, HSCs from a given clone had a consistent response to inflammatory or genotoxic stress
ORIGINAL ARTICLE Yu, V. W. C. et al. Epigenetic memory underlies cell-autonomous heterogeneous behavior of hematopoietic stem cells. Cell 167, 1310-1322 (2016) 